\title{
Plasmons in graphene nanoribbons: Interband transitions and nonlocal effects
}

\author{
Weihua Wang and Jari M. Kinaret* \\ Department of Applied Physics, Chalmers University of Technology, SE-412 96 Göteborg, Sweden \\ (Received 10 January 2013; revised manuscript received 20 March 2013; published 13 May 2013)
}

\begin{abstract}
We study plasmon excitations in infinitely long graphene nanoribbons using a quasistatic approach, where one-dimensional coupled equations for electrostatic potential and excited charge density are derived in the transverse direction. By incorporating a hydrodynamical description of the excited charge density, we investigate nonlocal effects in plasmon excitations. Moreover, the method presented here provides means to look into the nonlocal plasmon response in more complex graphene nanostructures such as wedges. We find that the plasmon frequencies are lowered by interband transitions and raised due to nonlocal effects. The frequency shifts depend monotonically on the dielectric constant of the surrounding medium. Most importantly, the nonlocal effects can strongly affect the excited charge density at the edges. Finally, we show that a larger increase of dipolar plasmon frequencies occurs in smaller graphene nanoribbons as expected.
\end{abstract}

DOI: 10.1103/PhysRevB.87.195424

PACS number(s): 73.20.Mf, 71.35.Ji, 78.67.Wj

\section{INTRODUCTION}

Graphene, which has been the subject of intense research since $2004,{ }^{1}$ is a one atom thin layer of carbon atoms arranged in a honeycomb lattice. ${ }^{2}$ It has been shown that graphene is a gapless semiconductor, for which the valence and conduction bands meet at the $\mathbf{K}$ and $\mathbf{K}^{\prime}$ points, and near these points the charge carriers obey a linear dispersion relation, thus acting as Dirac particles. ${ }^{3,4}$ The Fermi level in graphene can be easily adjusted by either electrostatic gating or chemical doping, ${ }^{5-7}$ which provides the means to flexibly control most of its electro-optical properties. Due to these unique characteristics, graphene is a promising material for a wide range of photonics and optoelectronics applications. ${ }^{8}$

Very recently, the rise of graphene plasmonics has attracted great interest in theoretical and experimental studies. ${ }^{9-12}$ A key advantage of graphene plasmonics is gate tunability, which has been confirmed by recent near-field optical microscopy experiments. ${ }^{13,14}$ Similar to metals, the large momentum mismatch between incident waves and plasmons can be overcome by engineering graphene nanostructures, such as ribbons ${ }^{15-20}$ and disks, ${ }^{21-24}$ which have been studied intensively. Since they are usually tens of nanometers or more in size, a local description of the optical conductivity $\left.\sigma(q, \omega)\right|_{q \rightarrow 0}$ is commonly used, where $\sigma(q, \omega)$ is obtained for an infinite graphene sheet using, e.g., the random phase approximation (RPA). ${ }^{9,10,25}$ However, at the nanoscale, significant plasmon blueshifts are seen due to nonlocal effects, ${ }^{18}$ and at the atomic scale nonlocal effects substantially influence plasmon resonances. ${ }^{26,27}$ Hence, it is necessary to explore nonlocal effects in plasmon excitations, in particular in wedges and sharp tips.

\section{HYDRODYNAMIC MODEL AND AN APPROXIMATE GRAPHENE CONDUCTIVITY}

In this article, we investigate plasmon excitations in graphene nanoribbons by using a quasistatic approach and study nonlocal effects by a hydrodynamic model for the excited charge density. The graphene nanoribbons are assumed to lie in the $x-y$ plane, with infinite length along the longitudinal $y$ direction and width $d$ in the transverse $x$ direction. Because of the translational invariance along the $y$ direction, all time dependent physical quantities have a common factor $e^{i(q y-\omega t)}$. Here $q$ is the momentum along the longitudinal direction and $\omega$ is the angular frequency of the waves.

The hydrodynamic model for graphene structures can be derived similarly as for other electron systems. It gives a connection between the in-plane currents $\mathbf{J}$ and electric fields $\mathbf{E}:^{28,29}$

$$
\mathbf{J}=\sigma(\omega) \mathbf{E}-\frac{\beta^{2}}{\omega^{2}} \nabla(\nabla \cdot \mathbf{J}),
$$

where $\sigma(\omega)$ is the local conductivity of the materials, and the $\beta^{2}$ term arises from the pressure in an inhomogeneous electron fluid. This equation can be rewritten as an expression for the in-plane charge density $\rho$ by taking divergence on both sides, thus yielding

$\beta^{2}\left[\frac{\partial^{2}}{\partial x^{2}}-q^{2}\right] \rho(x)+\omega^{2} \rho(x)=i \omega \sigma(\omega)\left[\frac{\partial^{2}}{\partial x^{2}}-q^{2}\right] \Phi(x)$,

where the continuity equation $-i \omega \rho+\nabla \cdot \mathbf{J}=0$ and $E=$ $-\nabla \Phi$ have been incorporated. The continuities of $\Phi$ and $\partial_{x} \Phi=0$ at edges are employed as the boundary conditions.

Equation (2) tells us that the charge density $\rho(x)$ is produced by the electrostatic potential $\Phi$. On the other hand, we know a variation of charge density, say $\rho(x) e^{i q y} \delta(z)$, produces an electrostatic potential according to the Coulomb law:

$\Phi(x, y, z)=\frac{1}{4 \pi \varepsilon} \int_{-d / 2}^{d / 2} d x^{\prime} \int_{-\infty}^{\infty} d y^{\prime} d z^{\prime} \frac{\rho\left(x^{\prime}\right) e^{i q y^{\prime}} \delta\left(z^{\prime}\right)}{r}$,

where $r=\sqrt{\left(x-x^{\prime}\right)^{2}+\left(y-y^{\prime}\right)^{2}+\left(z-z^{\prime}\right)^{2}}$, and $\varepsilon$ is the dielectric constant of the surrounding medium. The integration of the $z$ component is obvious and also can be analytically performed in the $y$ direction using ${ }^{30}$

$$
K_{0}(q|x|)=\int_{-\infty}^{\infty} \frac{d k}{2} \frac{e^{i k x}}{\sqrt{k^{2}+q^{2}}},
$$

where $K_{0}(x)$ is the zero-order modified Bessel function. The integration of Eq. (3) with respect to the argument $y^{\prime}-y$ at 
$z=0$ yields

$$
\Phi(x)=\frac{1}{2 \pi \varepsilon} \int_{-d / 2}^{d / 2} d x^{\prime} K_{0}\left(q\left|x-x^{\prime}\right|\right) \rho\left(x^{\prime}\right) .
$$

Combining Eqs. (2) and (5), we can study the local and nonlocal plasmon excitations in such a self-consistent way.

The nonlocal factor $\beta$ can be given from hydrodynamics theory at low frequencies; ${ }^{28,31,32}$ at higher frequencies, it should be replaced by a value that is consistent with the RPA expression for conductivity as suggested by Halevi. ${ }^{32}$ This approximation has been employed to investigate nonlocal effects on plasmon excitations in metals, ${ }^{29,33}$ where the conductivity of metals can be well described by the Drude model.

The nonlocal response has been shown to produce significant blueshifts of the plasmon frequencies at metallic nanostructures. ${ }^{34}$ However, for graphene the situation is somewhat more complicated: it has been demonstrated that plasmon frequencies exhibit a redshift within RPA. ${ }^{9,10}$ At highly charged graphene samples and within the longwavelength limit, to say $q \cdot v_{F}<<\omega<<E_{F}$, expanding the RPA conductivity for graphene $\sigma(q, \omega)$ in powers of $q$ gives

$$
\sigma(q, \omega)=\frac{e^{2} E_{F}}{\pi \hbar^{2}} \frac{i}{\omega}\left[1-\frac{\omega^{2}}{4 v_{F}^{2} k_{F}^{2}}+\frac{3 v_{F}^{2} q^{2}}{4 \omega^{2}}\right] .
$$

Here only the terms up to $q^{2}$ are kept, and we focus on the conceptually simplest uniform charge-density profile, which could be easy to achieve at chemically doped graphene, while at gated graphene, studied by S. Thongrattanasiri et al. $^{35}$ and Silvestrov and Efetov, ${ }^{36}$ the charge densities are extremely large at the edges. In the brackets, the first term is the classical Drude intraband contribution from conduction electrons, the second term is the interband contribution from valence electrons, and the third term is the nonlocal contribution from electron-electron Coulomb interaction. The same $q^{2}$ term correction has been demonstrated by Shung ${ }^{37}$ and Hill et al. ${ }^{38}$ We find that the expression of graphene conductivity has a similar form as that of a conventional two-dimensional electron system (2DES) except for the interband term. The interband term lowers the plasmon frequencies, as shown below, and the nonlocal term raises them, so that the net effect is a result of a competition between the two contributing factors. The opened gap at Dirac points is not considered here, ${ }^{39}$ which is much smaller than the Fermi energy studied in this article.

\section{NUMERICAL RESULTS AND DISCUSSION}

We proceed to study the plasmon excitations in graphene nanoribbons by solving the coupled equations (2) and (5). The high-frequency value of $\beta$ given by Eq. (6) is $\beta=\sqrt{3 / 4} v_{F}$, and the local conductivity $\sigma(\omega)$ in Eq. (1) includes the Drude and interband terms.

Without the nonlocal term, i.e., $\beta=0$ in Eq. (2), the coupled equations (2) and (5) can be solved by using the orthogonal polynomials expansion technique. ${ }^{23,24}$ Considering first the classical Drude conductivity only, the solutions of the eigenfrequencies $\omega_{n}(q)$ are a set of functions $a_{n}(q) \omega_{s}$, where the index $n$ corresponds to the number of nodes of each mode (charge-density distribution), and $\omega_{s}=\sqrt{e^{2} E_{F} /\left(2 \pi \hbar^{2} \varepsilon d\right)}$ is

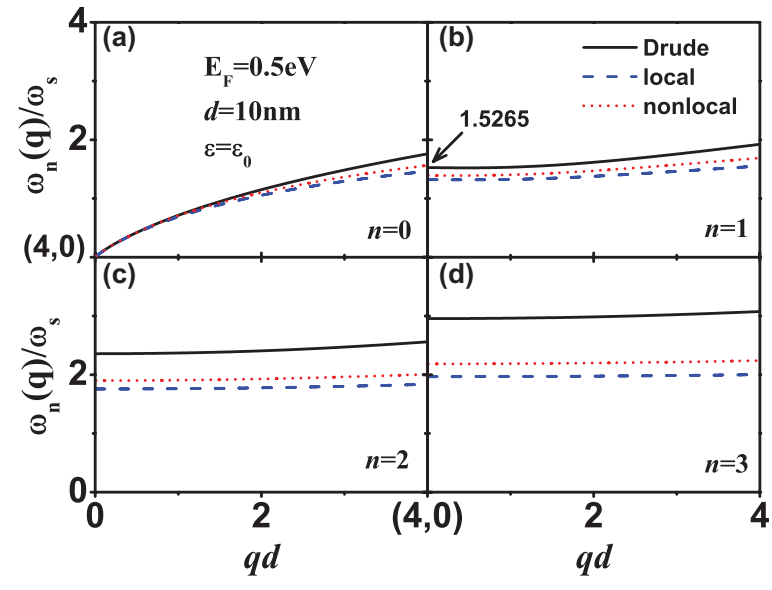

FIG. 1. (Color online) Plasmon dispersions of the first four modes for $E_{F}=0.5 \mathrm{eV}, d=10 \mathrm{~nm}$, and $\varepsilon=\varepsilon_{0}$. The black solid, blue dashed, and red short dashed curves represent the plasmon dispersion of the Drude model, local response, and nonlocal response, respectively.

the surface plasmon frequency at a wave vector equal to $1 / d$ in infinite graphene sheets. In addition, $a_{n}(q)$ only depend on the momentum $q$ but not on other physical parameters. This is the electrostatic scaling law addressed by Christensen et al. in graphene ribbons ${ }^{17}$ and also by us in graphene disks. ${ }^{24}$ Including the interband contributions, the plasmon dispersions become

$$
\omega_{n}(q)=\frac{a_{n}(q) \omega_{s}}{\sqrt{1+a_{n}^{2}(q) \omega_{s}^{2} /\left(4 v_{F}^{2} k_{F}^{2}\right)}} .
$$

In this procedure, the interband contributions only influence the plasmon frequencies. It is seen that the plasmon frequencies are reduced by an amount that depends on $a_{n}(q)$ and the ratio of $\hbar \omega_{s} / E_{F}$. Hence, interband transitions lead to a violation of the electrostatic scaling law mentioned above. However, the violation can be very small for a nanoribbon with large $\varepsilon d E_{F}$. As a rough estimate, for $E_{F}=0.5 \mathrm{eV}, d=50 \mathrm{~nm}$, and $\varepsilon=\varepsilon_{0}$, we have $\omega_{s} \approx 0.17 \mathrm{eV}$ and $\omega_{s}^{2} /\left(4 v_{F}^{2} k_{F}^{2}\right) \approx 0.03$. Therefore, the changes of the lower modes are a few percent.

In the general case, taking into account both interband and nonlocal effects, we solve the coupled equations numerically using the finite element method (FEM) solver COMSOL. The results are shown in Fig. 1, where the dispersions of the first four modes are presented as functions of longitudinal momentum $q$. Only the lowest mode shown in Fig. 1(a) depends strongly on $q$, and the other three are weakly $q$ dispersive. The three dispersions of the lowest mode are overlapping for small $q$ but exhibit deviations with $q$ increasing. Even with the nonlocal contributions, the plasmon frequencies (red short dashed curves) are lower than the Drude values (black solid lines) in the free standing case.

This situation can be changed by the screening effect raising from the substrate, where $\varepsilon$ is larger than $\varepsilon_{0}$. For example, for graphene on a silicon dioxide substrate, the average dielectric constant of the surrounding medium is given by $\varepsilon=\left(\varepsilon_{0}+\varepsilon_{\text {sub }}\right) / 2=2.5 \varepsilon_{0}$. The reduction of interband effects due to smaller $\omega_{s}$ is evident in Eq. (7), while the nonlocal 

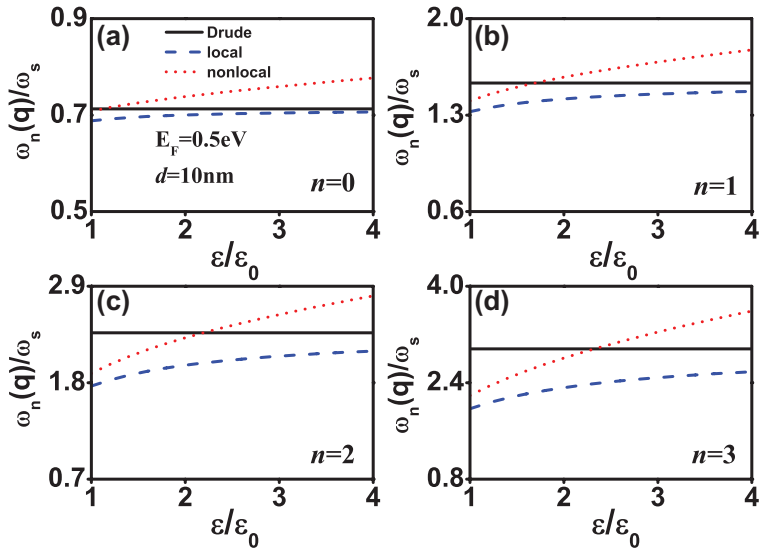

FIG. 2. (Color online) Plasmon frequencies for the first four modes as functions of $\varepsilon$ at $q=1 / d$ for $E_{F}=0.5 \mathrm{eV}$ and $d=10 \mathrm{~nm}$. The black solid, blue dashed, and red short dashed curves show the plasmonics frequencies of the Drude model, local response, and nonlocal response, respectively.

effects are largely unaffected by the substrate. In principle, there should be a crossing point, at which the two contributions are equal but opposite, and after that the nonlocal effects are larger than interband transitions.

This simple argument can be verified by numerically solving the coupled equations with varying $\varepsilon$. The solutions are plotted in Fig. 2. We see that the blue dashed curves approach the solid black lines as $\varepsilon$ increases, which indicates a lower contribution from interband transitions. The red short dashed curves (plasmon frequencies within nonlocal responses) are increasing continuously and have an intersection with that of the classical one. Moreover, the lower-energy mode can have the intersection at smaller $\varepsilon$, for instance, in this specific case, $\varepsilon=1.1 \varepsilon_{0}$ for $\omega_{0}(q)$ mode and $\varepsilon=1.7 \varepsilon_{0}$ for $\omega_{1}(q)$ mode.

The transverse charge-density profiles $\rho(x)$ for the first four modes are shown in Fig. 3. They are clearly distinguished by the number of nodes, which equals the mode index $n$. The total excited charge of any mode equals zero due to the
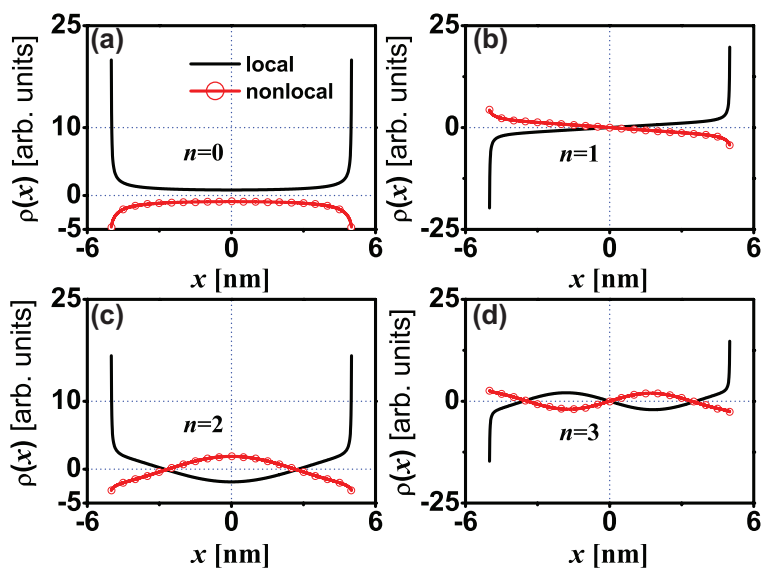

FIG. 3. (Color online) Excited charge density profile $\rho(x)$ for the first four modes at $q=1 / d$. The black solid lines and red circled lines are the charge-density profiles for local and nonlocal plasmon resonances, respectively.

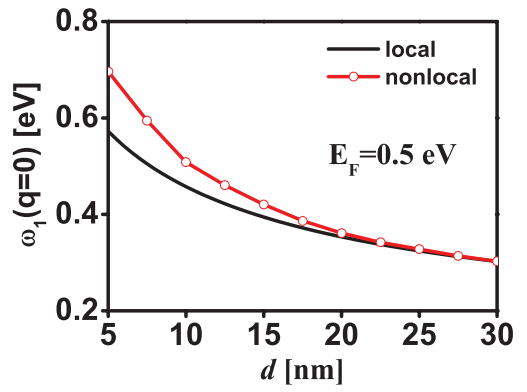

FIG. 4. (Color online) Plasmon frequencies of the dipolar mode as a function of width $d$ of the ribbon at $q=0$ for $E_{F}=0.5 \mathrm{eV}$ and $\varepsilon=\varepsilon_{0}$. The black solid and red circle curves are plasmon frequencies of local and nonlocal response, respectively.

sinusoidal variation in the $y$ direction. The lowest mode, with $\omega_{0}(q=0)=0$, is clearly confined to the edges. The other three modes have nodes in the transverse direction and depend weakly on the longitudinal momentum $q$; they are guided modes in the transverse direction and can be excited also at $q=0$. With the nonlocal term, the general profiles of plasmon modes do not change, but they become less strongly bound to the edges. This increases the importance of the bulk electrons and reduces the local-field enhancement at the edges due to the decrease of charge density there.

Of the four lowest modes, the second lowest mode $\left[\omega_{1}(q)\right.$, dipolar mode; see the charge density profile in Fig. 3(b)] is of particular interest, because it can strongly couple to an incident light. As mentioned above, it can also be excited at $q=0$. In order to study this special limit, we can use $K_{0}\left(q\left|x-x^{\prime}\right|\right) \rightarrow-\ln \left(\left|x-x^{\prime}\right|\right)$ in the integral equation (5). We find that the properties of the guided modes are very similar as in the $q \neq 0$ case, consistent with the fact that the guided modes depend only weakly on the longitudinal momentum $q$. The nonlocal effects introduce significant size dependency in plasmonic properties of sufficiently small structures. This is shown in Fig. 4, where we plot the frequencies of the $q=0$ dipolar plasmon mode for graphene ribbons as a function of the ribbon width. It is clearly seen that the nonlocal corrections are insignificant for wide ribbons but clearly visible for small structures. We notice that the nonlocal effects shown here are slightly larger than those found in Ref. [18], probably because of the approximations inherent in the hydrodynamic model. However, the trends and profiles show a good agreement, demonstrating the value of the simple approximation.

\section{CONCLUSIONS}

In this article, we have studied the plasmon properties in graphene nanoribbons beyond the quasistatic approach. The nonlocal effects have been investigated using a hydrodynamical description of the excited charge density. We find that there is a competition between the interband transitions and nonlocal effects which result in lowering and raising of the plasmon frequencies, respectively. The relative importance of two contributions can be changed by the substrate, and the strong screening can reduce the interband contribution. Including the nonlocal effects, plasmons show a similar excited charge-density profile as in the Drude limit, but the charge 
densities are less bound to the edges. The dipolar plasmon is seen to exhibit substantial blueshifts in narrow graphene ribbons, demonstrating the importance of nonlocal corrections in graphene nanostructures.

\section{ACKNOWLEDGMENTS}

We thank S. Peter Apell for stimulating discussion. This work was supported by the Swedish Research Council (VR). *jari.kinaret@chalmers.se

${ }^{1}$ K. S. Novoselov, A. K. Geim, S. V. Morozov, D. Jiang, Y. Zhang, S. V. Dubonos, I. V. Grigorieva, and A. A. Firsov, Science 306, 666 (2004).

${ }^{2}$ A. K. Geim and K. S. Novoselov, Nat. Mater. 6, 183 (2007).

${ }^{3}$ A. H. Castro Neto, F. Guinea, N. M. R. Peres, K. S. Novoselov, and A. K. Geim, Rev. Mod. Phys. 81, 109 (2009).

${ }^{4}$ S. Das Sarma, S. Adam, E. H. Hwang, and E. Rossi, Rev. Mod. Phys. 83, 407 (2011).

${ }^{5}$ A. K. Geim, Science 324, 1530 (2009).

${ }^{6}$ K. F. Mak, M. Y. Sfeir, Y. Wu, C. H. Lui, J. A. Misewich, and T. F. Heinz, Phys. Rev. Lett. 101, 196405 (2008).

${ }^{7}$ C.-F. Chen, C.-H. Park, B. W. Boudouris, J. Horng, B. Geng, C. Girit, A. Zettl, M. F. Crommie, R. A. Segalman, S. G. Louie, and F. Wang, Nature (London) 471, 617 (2011).

${ }^{8}$ F. Bonaccorso, Z. Sun, T. Hasan, and A. Ferrari, Nature Photonics 4, 611 (2010).

${ }^{9}$ E. H. Hwang and S. Das Sarma, Phys. Rev. B 75, 205418 (2007).

${ }^{10}$ M. Jablan, H. Buljan, and M. Soljacic, Phys. Rev. B 80, 245435 (2009).

${ }^{11}$ F. H. L. Koppens, D. E. Chang, and F. J. García de Abajo, Nano. Lett. 11, 3370 (2011).

${ }^{12}$ Z. Fei, G. O. Andreev, W. Bao, L. M. Zhang, A. S. McLeod, C. Wang, M. K. Stewart, Z. Zhao, M. Dominguez, Gerardo Thiemens, M. M. Fogler, M. J. Tauber, A. H. Castro-Neto, C. N. Lau, F. Keilmann, and D. N. Basov, Nano. Lett. 11, 4701 (2011).

${ }^{13}$ J. Chen, M. Badioli, P. Alonso-González, S. Thongrattanasiri, F. Huth, J. Osmond, M. Spasenović, A. Centeno, A. Pesquera, P. Godignon, A. Z. Elorza, N. Camara, F. J. García de Abajo, R. Hillenbrand, and F. H. L. Koppens, Nature (London) 487, 77 (2012).

${ }^{14}$ Z. Fei, A. S. Rodin, G. O. Andreev, W. Bao, A. S. McLeod, M. Wagner, L. M. Zhang, Z. Zhao, M. Thiemens, G. Dominguez, M. M. Fogler, A. H. Castro Neto, C. N. Lau, F. Keilmann, and D. N. Basov, Nature (London) 487, 82 (2012).

${ }^{15}$ E. G. Mishchenko, A. V. Shytov, and P. G. Silvestrov, Phys. Rev. Lett. 104, 156806 (2010).

${ }^{16}$ L. Ju, B. Geng, J. Horng, C. Girit, M. Martin, Z. Hao, H. A. Bechtel, X. Liang, A. Zettl, Y. R. Shen, and F. Wang, Nature Nanotechnology 6, 630 (2011).

${ }^{17}$ J. Christensen, A. Manjavacas, S. Thongrattanasiri, F. H. L. Koppens, and F. J. García de Abajo, ACS Nano 6, 431 (2011).
${ }^{18}$ S. Thongrattanasiri, A. Manjavacas, and F. J. García de Abajo, ACS Nano 6, 1766 (2012).

${ }^{19}$ A. Y. Nikitin, F. Guinea, F. J. García-Vidal, and L. Martín-Moreno, Phys. Rev. B 84, 161407 (2011).

${ }^{20}$ A. Y. Nikitin, F. Guinea, F. J. Garcia-Vidal, and L. Martin-Moreno, Phys. Rev. B 85, 081405 (2012).

${ }^{21}$ S. Thongrattanasiri, F. H. L. Koppens, and F. J. García de Abajo, Phys. Rev. Lett. 108, 047401 (2012).

${ }^{22}$ H. Yan, X. Li, B. Chandra, G. Tulevski, Y. Wu, M. Freitag, W. Zhu, P. Avouris, and F. Xia, Nature Nanotechnology 7, 330 (2012).

${ }^{23}$ W. Wang, P. Apell, and J. Kinaret, Phys. Rev. B 84, 085423 (2011).

${ }^{24}$ W. Wang, S. P. Apell, and J. M. Kinaret, Phys. Rev. B 86, 125450 (2012).

${ }^{25}$ B. Wunsch, T. Stauber, F. Sols, and F. Guinea, New J. Phys. 8, 318 (2006).

${ }^{26}$ J. Yan, K. S. Thygesen, and K. W. Jacobsen, Phys. Rev. Lett. 106, 146803 (2011).

${ }^{27}$ W. Zhou, J. Lee, J. Nanda, S. T. Pantelides, S. J. Pennycook, and J.-C. Idrobo, Nature Nanotechnology 7, 161 (2012).

${ }^{28}$ J. M. Pitarke, V. M. Silkin, E. V. Chulkov, and P. M. Echenique, Rep. Prog. Phys. 70, 1 (2007).

${ }^{29}$ S. Raza, G. Toscano, A.-P. Jauho, M. Wubs, and N. A. Mortensen, Phys. Rev. B 84, 121412 (2011).

${ }^{30}$ Handbook of Mathematical Functions with Formulas, Graphs, and Mathematical Tables, edited by M. Abramowitz and I. A. Stegun (U.S. GPO, Washington, DC, 1964).

${ }^{31} \mathrm{~S}$. Lundqvist, in Theory of the Inhomogeneous Electron Gas, edited by S. Lundqvist and N. H. March (Plenum, New York, 1983).

${ }^{32}$ P. Halevi, Phys. Rev. B 51, 7497 (1995).

${ }^{33}$ A. I. Fernández-Domínguez, A. Wiener, F. J. García-Vidal, S. A. Maier, and J. B. Pendry, Phys. Rev. Lett. 108, 106802 (2012).

${ }^{34}$ F. J. García de Abajo, J. Phys. Chem. C 112, 17983 (2008).

${ }^{35}$ S. Thongrattanasiri, I. Silveiro, and F. J. García de Abajo, Appl. Phys. Lett. 100, 201105 (2012).

${ }^{36}$ P. G. Silvestrov and K. B. Efetov, Phys. Rev. B 77, 155436 (2008).

${ }^{37}$ K. W.-K. Shung, Phys. Rev. B 34, 979 (1986).

${ }^{38}$ A. Hill, S. A. Mikhailov, and K. Ziegler, Europhys. Lett. 87, 27005 (2009).

${ }^{39}$ Y.-W. Son, M. L. Cohen, and S. G. Louie, Phys. Rev. Lett. 97, 216803 (2006) 